\title{
The Effect of Warfarin Administration Time on Anticoagulation Stability (INRange): A Pragmatic Randomized Controlled Trial
}

Scott R. Garrison, $M D, P b D^{1}$

Lee Green, $M D, M P H^{1}$

Michael R. Kolber, MD, MSc'

Christina S. Korownyk, MD

Nicole M. Olivier, RVT

Balraj S. Heran, PbD

Mary E. Flesher, RD, $M A^{3}$

G. Michael Allan, $M D^{1}$

'Department of Family Medicine, University of Alberta, Edmonton, Alberta, Canada

${ }^{2}$ Department of Anesthesiology, Pharmacology and Therapeutics, University of British Columbia, Vancouver, British

Columbia, Canada

${ }^{3}$ Fraser Health Authority, Burnaby, British Columbia, Canada

Conflicts of interest: authors report none

\section{CORRESPONDING AUTHOR}

Scott R. Garrison, MD, PhD

Evidence Based Medicine

6-60 University Terr

Edmonton, Alberta

Canada T6G 2T4

scott.garrison@ualberta.ca

\begin{abstract}
PURPOSE Without supporting evidence, clinicians commonly recommend that warfarin be taken in the evening. We conducted a randomized controlled trial to evaluate the effect of administration time (morning vs evening) on the stability of warfarin's anticoagulant effect.
\end{abstract}

METHODS A total of 236 primary care physicians serving 54 western Canadian communities mailed letters of invitation to all their warfarin-using patients. Eligible patients were community-dwelling warfarin users (any indication) with at least 3 months of evening warfarin use and no plans for discontinuation. Participants were randomized (by web-based allocation) to morning vs continued evening warfarin ingestion. We used the Rosendaal method to determine the proportion of time within therapeutic range (TTR) of the international normalized ratio (INR) blood test month 2 to 7 postrandomization vs the 6 months prerandomization. The primary outcome was the percent change in proportion of time outside target INR range (with an a priori minimum clinically important difference of $\pm 20 \%)$. All analyses were intention to treat.

RESULTS Between March 8, 2015 and September 30, 2016, we randomized 109 participants to morning and 108 to evening warfarin use. TTR rose from $71.8 \%$ to $74.7 \%$ in the morning group, and from $72.6 \%$ to $75.6 \%$ in the evening group, for a change in TTR of $2.9 \%$ in the former vs $3.0 \%$ in the latter (difference, $-0.1 \% ; P=.97 ; 95 \% \mathrm{Cl}$ for the difference, $-6.1 \%$ to $5.9 \%)$. The difference in percent change in proportion of time outside the therapeutic INR range (obtained via Hodges-Lehmann estimation of the difference in medians) was $4.4 \%(P=.62 ; 95 \% \mathrm{Cl}$ for the difference, $-17.6 \%$ to $27.3 \%)$.

CONCLUSIONS Administration time has no statistically significant nor clinically important impact on the stability of warfarin's anticoagulant effect. Patients should take warfarin whenever regular compliance would be easiest.

Ann Fam Med 2020;18:42-49. https://doi.org/10.1370/afm.2488.

\section{INTRODUCTION}

0 troke and pulmonary embolism have devastating, often lifelong health consequences, and conditions that predispose to these events (atrial fibrillation, deep vein thrombosis, and mechanical heart valves) are common. Warfarin substantially reduces the risk of such thromboembolic events. ${ }^{1}$ The safety and effectiveness of warfarin depends greatly on the proportion of time in the therapeutic range (TTR) of the international normalized ratio (INR) blood test, however. ${ }^{2-5}$

Most physicians and pharmacists recommend warfarin be taken in the early evening. ${ }^{6-10}$ This strategy likely shortens the interval between learning of the need to make a dose adjustment (typically communicated to patients in the late afternoon following a morning blood test) and being able to make that dosing change. Hence, if evening warfarin use means quicker dose adjustments, it might conceivably lead to better TTR. Although the hypothesis is reasonable, there is no evidence to support this practice and other factors 
could meaningfully influence optimal administration time. For instance, dietary vitamin $\mathrm{K}$ (with which warfarin interacts) has an ultrashort 2.5-hour half-life and is found in foods (green leafy vegetables) having highly variable intake and rarely ingested in the morning. ${ }^{11,12}$ Conceivably, ingesting warfarin in the morning, when vitamin $\mathrm{K}$ levels are more consistent, might lead to greater INR stability. Although patients are commonly advised to take warfarin in the evening, it is unclear whether administration time matters, and if it does, which time is best.

In this pragmatic primary care study, The Effect of Warfarin Administration Time on Anticoagulation Stability (INRange), we randomized established warfarin users taking the medication in the evening either to switch to morning warfarin use or to continue evening use, and examined the TTR to detect differences in the stability of warfarin's anticoagulant effect.

\section{METHODS}

\section{Study Design and Setting}

INRange was a prospective randomized, open, blindedendpoint (PROBE) ${ }^{13}$ study carried out in the offices of 236 primary care clinicians serving 54 western Canadian communities. Most clinicians were community family physicians in full-time, fee-for-service practice who were practicing remotely from academic centers but affiliated with the Pragmatic Trials Collaborative ${ }^{14}$ (a multiprovincial practice-based research network). Patient participants were recruited with letters of invitation and study information packages that their primary care clinicians mailed to all warfarin users under their care. Interested patients called a contact number in the information package and were assessed by telephone for eligibility by a research assistant. A detailed study protocol and analytic plan have been published. ${ }^{15}$ No alterations to the registered or published protocol were made.

\section{Participants}

Trial inclusion criteria were (1) dinner or evening warfarin use ${ }_{i}(2)$ community-dwelling status (assisted living residents were allowed to participate, but only if they had control of their own medication timing); (3) an expectation of long-term warfarin use; ${ }_{i}(4)$ availability of at least 3 months of baseline INR data (the last 6 months were used if available) with at least 4 evaluable INR results no more than 8 weeks apart from another INR reading.

Although no formal exclusion criteria were applied when patients were assessed for eligibility, clinicians were asked not to mail invitation letters to those they believed to have a limited life expectancy (less than 1 year) or to be incapable of providing informed consent.

All participants provided informed consent, either in writing or online.

\section{Randomization and Masking}

When the research team obtained adequate baseline INR data from the primary care clinician, consented eligible participants received a telephone call from a research assistant (with no clinical interactions) who obtained the participant's allocation group over the web from the central research electronic data capture (REDCap) ${ }^{16}$ server's randomization module (ensuring irreversible and concealed allocation). To minimize imbalance, the randomization sequence was stratified by percentage of readings within therapeutic range $(<50 \%, 50 \%$ to $80 \%,>80 \%)$ and used variable blocks of 2 or 4 . Study evaluators were blinded to allocation, but patients (who administered their own medications) were not blinded and free to share this information with their clinicians if either deemed it clinically relevant.

\section{Procedures}

This study was prospectively registered with ClinicalTrials.gov as NCT02376803 on February 25,2015 , before any patients were enrolled. Ethics approval was obtained from the clinical research ethics boards at the University of Alberta, and the University of British Columbia.

\section{Data Collection}

Baseline characteristics and information believed predictive of TTR were obtained directly from patients during a telephone interview immediately before randomization. These data included the self-reported average number of days per week that foods having a high vitamin $\mathrm{K}$ content (kale, spinach, chard, beet greens, broccoli, Brussels sprouts, romaine lettuce, or cabbage) were consumed, and how variable the participant felt this estimated level of vitamin K consumption was on a 4 -point scale.

For follow-up, participants could choose between e-mail questionnaires generated by REDCap or telephone interviews, occurring 1 week, 1 month, and 7 months after either their timing change if they were in the intervention group (with the change made 5 days before the next scheduled INR test) or the date of randomization if they were continuing with evening use. During these interviews, participants self-reported compliance with allocation, as well as any illnesses and potentially warfarin-related adverse events (bleeding and thromboembolic events). Follow-up INR data for the 7 months postrandomization were obtained directly from primary care clinicians.

\section{Warfarin Management}

Patients' clinicians continued to manage their warfarin therapy as per their usual routine, with no initial changes to dosing or any planned initial changes to the 
frequency of INR testing. In 152 of the 236 practices mailing out invitations, warfarin was managed solely by a family physician. In the remaining 84 practices warfarin was managed by clinic-affiliated nurses (74 practices) and pharmacists (10 practices), with consultation of the family physician as required.

\section{Outcomes}

The primary outcome was the percent change in the proportion of time spent outside the target INR range, with the minimum clinically important difference predefined to be $\pm 20 \%$. (The rationale for selecting this value and others is outlined below.) Secondary outcomes were the absolute change in TTR, the percent of patients with TTR above and below various cutoffs, the maximum and minimum out-of-range INR values, and the percent of INR values above and below the range.

As an exploratory outcome, we assessed for an interaction between warfarin timing and a patient's self-assessment of the variability and number of days per week that foods with high vitamin K content were consumed in any amount.

All outcomes were as described in the registered and published protocols with no alterations.

\section{Statistical Analyses}

\section{Minimum Clinically Important Difference}

For patients with atrial fibrillation, an estimated 7\% absolute improvement in TTR would prevent 1 major hemorrhage per 100 patient-years. ${ }^{4}$ Over the same period, an estimated $12 \%$ absolute improvement would prevent 1 thromboembolic event. ${ }^{4}$ These numbers are comparable to the $5 \%$ to $10 \%$ minimum clinically important difference for absolute change in TTR that was suggested by an informal sampling of clinicians, ${ }^{17}$ and are consistent with the statement from a randomized controlled trial that an observed (statistically significant) $6 \%$ absolute difference in TTR was "modest" and "less than the minimum clinically important difference" they had predefined (10\%). ${ }^{18}$ We therefore chose $6 \%$ as our minimum clinically important difference for absolute change in TTR. A trial similar to ours (a Canadian primary care randomized controlled trial recruiting all warfarin users) reported a 70\% baseline TTR. ${ }^{19}$ Expecting similar baseline characteristics (ie, that participants would be out of range $30 \%$ of the time at baseline), the corresponding minimum clinically important difference for percent change in proportion of time out of range was thus $6 \%$ out of $30 \%$ (ie, $\pm 20 \%$ ).

\section{Sample Size}

We determined that 170 participants (increased to 200 to account for potential dropouts and noncompliance) were needed to demonstrate a $20 \%$ difference in the percent change in proportion of time outside therapeutic INR range, assuming $90 \%$ power, an alpha error of .05 , and a standard deviation twice the mean effect (ie, $\mathrm{SD}=40 \%)$.

\section{Calculating TTR}

Target INR ranges vary by patient and by physician. Typically, the therapeutic range is 1 unit wide, usually either 2 to 3 , or 2.5 to 3.5 depending on the indication for anticoagulation. It can, however, sometimes be narrower or wider (eg, 2 to 2.5 , or 2 to 3.5 ). To standardize the width of each patient's target INR range, we determined the midpoint of their individual target range and set their standardized target range to be 0.5 units above and below this midpoint (eg, a wider 2 to 3.5 target, with a midpoint of 2.75 , would translate to a standardized target range of 2.25 to 3.25 ).

\section{Primary and Secondary Analyses}

All analyses were by intention to treat. If follow-up data were missing or insufficient, we assigned them the baseline value (ie, assumed no change). The primary outcome, percent change in proportion of time outside therapeutic INR range, could be analyzed only in patients for whom the proportion of time outside therapeutic range was nonzero at baseline. This situation occurs because the percent change in anything is not calculable when its baseline value is zero (since you cannot divide by zero). Hence, we assigned all patients who were never out of range at baseline the smallest observed nonzero baseline value (ie, treated them as being out of range $0.77 \%$ of the time). All statistical analyses were completed using PRISM 7.0d software (GraphPad Software) and all $P$ values were 2 -sided.

\section{Comparison of Outcomes}

For continuous outcomes, when comparisons of change from baseline were normally distributed, we used the difference in sample means to estimate effect size, and the Student $t$ test to determine statistical significance. When outcomes were not normally distributed, statistical significance was determined by the Mann-Whitney U test and the Hodges-Lehmann estimate for difference in medians was used to determine effect size.

For dichotomous outcomes, the Fisher exact test was used to compare values, with confidence intervals calculated using the Koopman asymptotic score (for relative risk) and the Newcombe-Wilson score with continuity correction (for attributable risk).

\section{Sensitivity Analysis}

The percent change in the proportion of time that patients are out of range can have large swings when 
anticoagulation status is very well controlled. For example, if a patient has a $98 \%$ baseline TTR that falls to $90 \%$, their proportion of time out of range 0.02 rising to 0.1 ) rises by $400 \%$. The subset of patients with initially very well controlled anticoagulation could therefore disproportionately drive the analysis. To account for this possibility, we performed a sensitivity analysis for the primary outcome including only patients who were out of range more than $10 \%$ of the time (ie, having a TTR <90\%).

\section{Exploratory Analysis}

A Kruskal-Wallis test (1-way ANOVA of ranks) was used to search for an interaction between warfarin timing and 2 patient-reported measures: average number of days per week that foods having high vitamin $\mathrm{K}$ content were consumed $(<2$ days, 2-5 days, >5 days), and variability in the consumption of those foods (a binary high or low variable constructed from 4 possible responses).

\section{Data Availability}

At the time of publication of this article, anonymized patient-level data, including all nonidentifying baseline characteristics and outcomes, will be made available to the public at http://www. PragmaticTrials.ca.

\section{RESULTS}

The study took place between March 8, 2015, and September 30, 2016, with 236 primary care physicians in 54 western Canadian communities mailing 2,107 recruitment letters. We assessed 351 patients for eligibility and excluded 134 before randomization (Figure 1). Of these, 93 were excluded because they already used warfarin in the morning, 10 were reluctant to change medication timing if randomized to do so, and 10 had baseline INR data that were either insufficient or unavailable. Other reasons for exclusion are shown in the trial flow diagram.

Of the 217 participants $(10.3 \%$ of those to whom letters were mailed), 109 were randomized to switch to morning warfarin and 108 we randomized to continue evening use as a control. The total exceeded the sam-
Figure 1. CONSORT flow diagram.

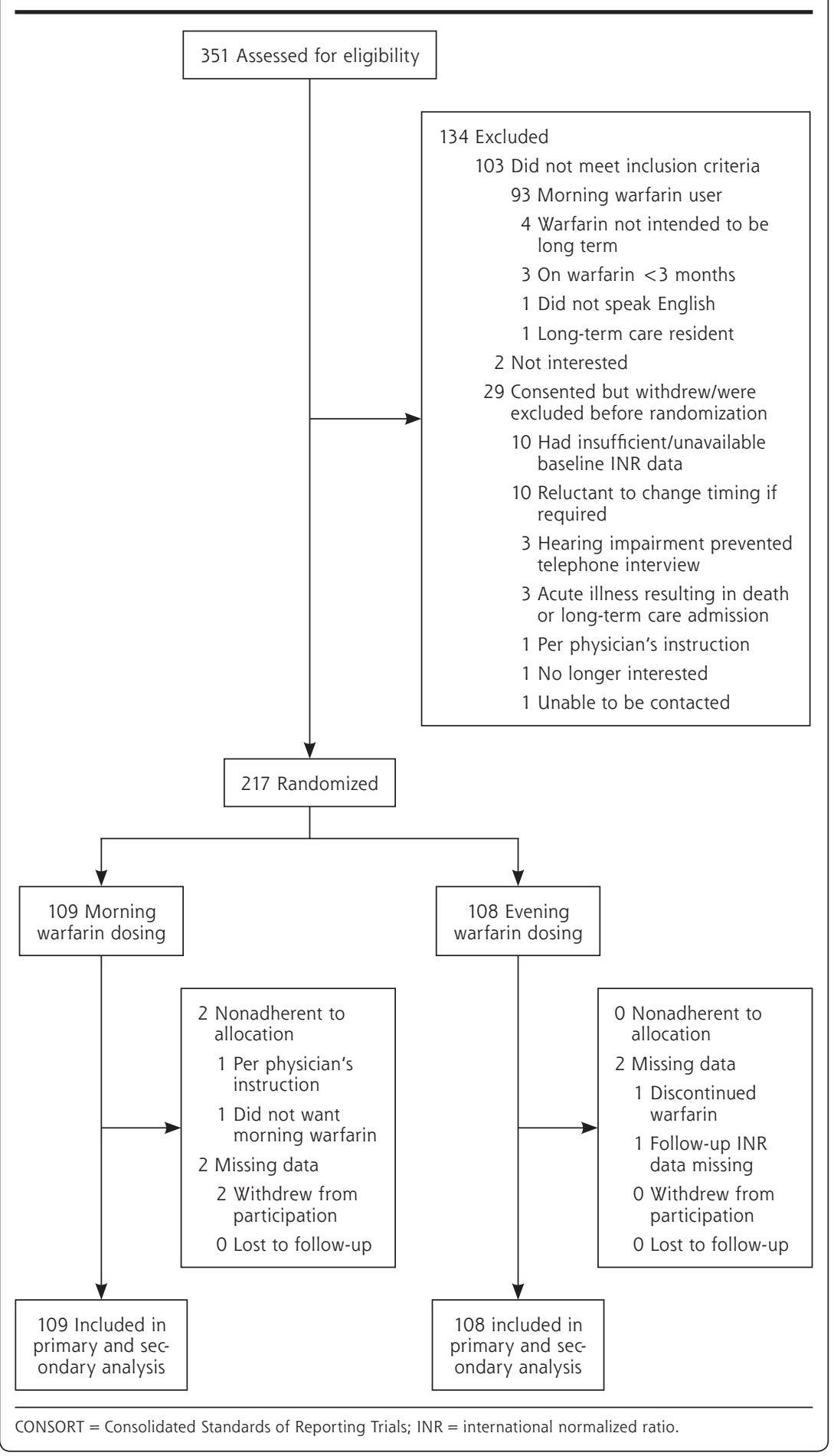


Table 1. Patients' Baseline Characteristics by Treatment Group

\begin{tabular}{|c|c|c|c|c|c|}
\hline Characteristic & $\begin{array}{l}\text { Morning } \\
(\mathrm{n}=109)\end{array}$ & $\begin{array}{l}\text { Evening } \\
(\mathrm{n}=108)\end{array}$ & Characteristic & $\begin{array}{l}\text { Morning } \\
(\mathrm{n}=109)\end{array}$ & $\begin{array}{l}\text { Evening } \\
(n=108)\end{array}$ \\
\hline \multicolumn{3}{|l|}{ Sex, No. (\%) } & \multirow{2}{*}{\multicolumn{3}{|c|}{$\begin{array}{l}\text { Stability of anticoagulation } \\
\text { (continued) }\end{array}$}} \\
\hline Male & $60(55.0)$ & $51(47.2)$ & & & \\
\hline Female & $49(45.0)$ & $57(52.8)$ & $\begin{array}{l}\text { Maximum out of range INR, } \\
\text { mean (SD) }\end{array}$ & $3.9(1.0)$ & $4.0(1.0)$ \\
\hline \multicolumn{3}{|l|}{ Age } & \multirow{2}{*}{\multicolumn{3}{|c|}{$\begin{array}{l}\text { Minimum out of range INR, } \\
\text { mean (SD) }\end{array}$}} \\
\hline Mean (SD), y & $72.7(10.3)$ & $73.4(10.3)$ & & & \\
\hline \multicolumn{3}{|l|}{ Province, No. (\%) } & $\begin{array}{l}\text { Percent of readings above range, } \\
\text { mean (SD) }\end{array}$ & $14.0(13.3)$ & $14.1(13.9)$ \\
\hline Alberta & $81(74.3)$ & $85(78.7)$ & $\begin{array}{l}\text { Percent of readings below range, } \\
\text { mean (SD) }\end{array}$ & $18.0(16.9)$ & $17.4(16.7)$ \\
\hline $\begin{array}{l}\text { British Columbia } \\
\text { Location, No. (\%) }\end{array}$ & $28(25.7)$ & $23(21.3)$ & \multicolumn{3}{|l|}{$\begin{array}{l}\text { Randomization stratification, } \\
\text { No. }(\%)\end{array}$} \\
\hline Urban & $86(78.9)$ & $96(88.9)$ & $<50 \%$ INR values in range & $17(15.6)$ & $17(15.7)$ \\
\hline Rural & $23(21.1)$ & $12(11.1)$ & $50 \%-80 \%$ INR values in range & $61(56.0)$ & $61(56.5)$ \\
\hline \multicolumn{3}{|l|}{$\begin{array}{l}\text { Indication for anticoagulation, } \\
\text { No. (\%) }\end{array}$} & $\begin{array}{l}>80 \% \text { INR values in range } \\
\text { Days per week of high-vitamin } \mathrm{K}\end{array}$ & $31(28.4)$ & $30(27.8)$ \\
\hline Atrial fibrillation & $68(62.4)$ & $63(58.3)$ & \multicolumn{3}{|l|}{$\begin{array}{l}\text { Days per week of high-vitamin K } \\
\text { foods, No. (\%) }\end{array}$} \\
\hline DVT/PE & $31(28.4)$ & $23(21.3)$ & $<2$ days & $44(40.4)$ & $30(27.8)$ \\
\hline Mechanical valve & $11(10.1)$ & $12(11.1)$ & 2-5 days & $47(43.1)$ & $57(52.8)$ \\
\hline Other & $10(9.2)$ & $17(15.7)$ & $>5$ days & $18(16.5)$ & $21(19.4)$ \\
\hline \multicolumn{3}{|l|}{ Target INR range, No. (\%) } & \multicolumn{3}{|l|}{$\begin{array}{l}\text { Variability of high-vitamin K } \\
\text { foods, No. (\%) }\end{array}$} \\
\hline $2.5-3.5$ & $7(6.4)$ & $11(10.2)$ & High & $44(40.4)$ & $48(44.4)$ \\
\hline Other & $5(4.6)$ & $0(0.0)$ & Low & $65(59.6)$ & $60(55.6)$ \\
\hline \multicolumn{3}{|l|}{ Warfarin use } & \multicolumn{3}{|l|}{ Medications other than warfarin } \\
\hline Daily dose, mean (SD), mg & $5.0(2.1)$ & $4.9(2.2)$ & \multicolumn{3}{|l|}{ Number, mean (SD) } \\
\hline Duration of use, mean (SD), y & $7.9(7.2)$ & $7.4(6.8)$ & Prescription medications & $4.8(3.4)$ & $5.0(3.3)$ \\
\hline$<6$ months of use, No. (\%) & $2(1.8)$ & $4(3.7)$ & Over-the-counter medications & $0.6(1.1)$ & $0.6(1.1)$ \\
\hline $\begin{array}{l}\text { Forget warfarin at least once per } \\
\text { month, No. }(\%)\end{array}$ & $8(7.3)$ & $8(7.4)$ & \multicolumn{3}{|l|}{ Prescription medications, No. (\%) } \\
\hline \multirow{2}{*}{\multicolumn{3}{|c|}{$\begin{array}{l}\text { Warfarin administration time, } \\
\text { No. }(\%)\end{array}$}} & Cardiovascular & $90(82.6)$ & $94(87.0)$ \\
\hline & & $18(16.7)$ & Gastrointestinal & $32(29.4)$ & $42(38.9)$ \\
\hline$\leq 30$ minutes after dinner & $46(42.2)$ & $49(454)$ & Diabetes & $14(12.8)$ & $21(19.4)$ \\
\hline$>30$ minutes after dinner & $\begin{array}{l}40(42.2) \\
13(11.9)\end{array}$ & $\begin{array}{c}49(43.4) \\
5(4.6)\end{array}$ & Pulmonary & $23(21.1)$ & $14(12.8)$ \\
\hline Bedtime & $32(29.4)$ & $35(32.4)$ & \multicolumn{3}{|l|}{ Antiplatelet agents, No. (\%) } \\
\hline Varies & $3(2.8)$ & 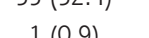 & Aspirin & $23(21.1)$ & $25(23.1)$ \\
\hline \multirow{2}{*}{\multicolumn{3}{|c|}{ Stability of anticoagulation }} & Clopidogrel & $2(1.8)$ & $5(4.6)$ \\
\hline & & & Prasugrel & $1(0.9)$ & $0(0.0)$ \\
\hline $\begin{array}{l}\text { Proportion of time within therapeu- } \\
\text { tic range of INR (TTR), mean (SD) }\end{array}$ & $71.8(20.2)$ & $72.6(19.5)$ & Dipyridamole/ticlopidine/ticagrelor & $0(0.0)$ & $0(0.0)$ \\
\hline $\begin{array}{l}\text { Proportion of time outside of thera- } \\
\text { peutic range of INR, mean (SD) }\end{array}$ & $28.2(20.2)$ & $27.2(19.6)$ & & & \\
\hline TTR >75\%, No. (\%) & $53(48.6)$ & $57(52.8)$ & & & \\
\hline TTR <60\%, No. (\%) & $34(31.2)$ & $24(22.2)$ & & & \\
\hline
\end{tabular}

ple size of 200 needed to demonstrate our 20\% minimum clinically important difference. Patients' baseline characteristics are shown in Table 1. For the intervention group, the rate of nonadherence to allocation (2 patients) and missing data ( 2 patients) was $3.7 \%$ combined, as compared with $1.9 \%$ in the control group.

The percent change in the proportion of time patients were outside therapeutic INR range was not normally distributed (D'Agostino-Pearson normality tests <.0001). The Hodges-Lehmann estimator for the difference in medians (morning vs evening) was $4.4 \%$ $(P=.62 ; 95 \% \mathrm{CI}$ for the difference, $-17.6 \%$ to $27.3 \%)$ with actual medians of $-22.9 \%$ vs $-11.9 \%$. This $4.4 \%$ difference was substantially less than our predefined $\pm 20 \%$ minimum clinically important difference.

The absolute change in TTR was normally distributed (Figure 2), with a value of $2.9 \%$ for the morning group vs $3.0 \%$ for the evening group $(P=.97 ; 95 \%$ CI for the difference, $-6.1 \%$ to $5.9 \%)$. Maximum and minimum INR, and percent of readings above and 
Figure 2. Scatterplot of absolute change in TTR.

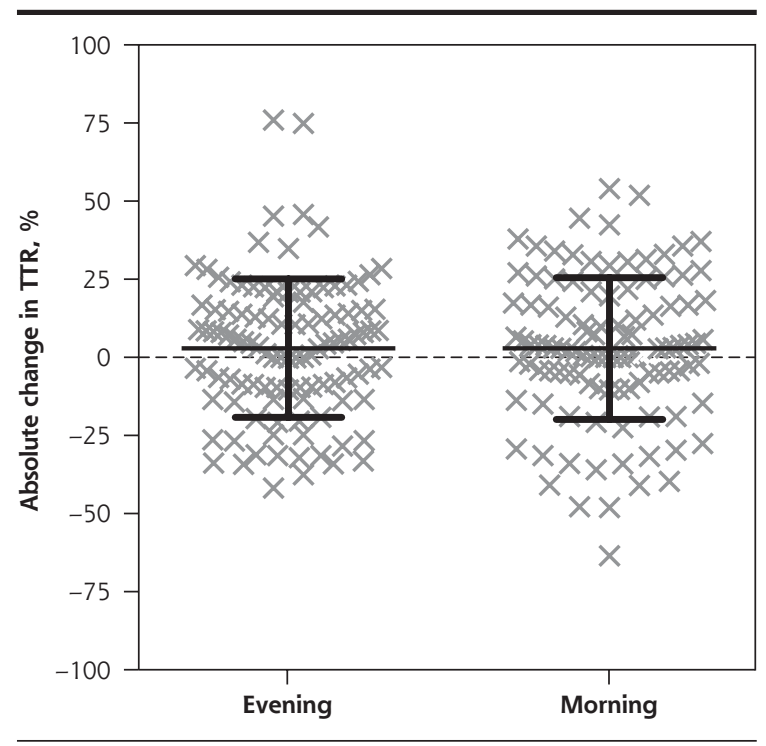

TTR = time within therapeutic range (of the international normalized ratio). Note: Each of the 217 data points represents the absolute change in TTR for 1 study participant. The overlaid black crossbars indicate mean and SD.

below therapeutic range (Table 2) were not normally distributed.

Major warfarin-related adverse cardiovascular events in the morning group included 1 gastrointestinal bleeding event, 1 thromboembolic stroke, and 1 deep vein thrombosis. These numbers compared with 1 thromboembolic stroke and 1 deep vein thrombosis in the evening group (who also had the only other acute arterial occlusion, a myocardial infarction).

Neither the self-reported number of days per week that foods having high vitamin $\mathrm{K}$ content were consumed $(P=.79)$ nor the patient's global estimate of the variability of those foods within the $\operatorname{diet}(P=.72)$ influenced the effect of the intervention on the primary outcome.

In our sensitivity analysis comparing the primary outcome in the 84 morning group and 85 evening group participants with baseline TTR of less than $90 \%$, the Hodges-Lehmann difference in medians, which now trended in the opposite direction, was $-5.3 \%$ $(P=.49 ; 95 \% \mathrm{CI}$ for the difference, $-25.8 \%$ to $11.3 \%)$.

\section{DISCUSSION}

Our results show that warfarin administration time, morning vs evening, has no clinically important effect on the proportion of time that warfarin users spend outside the therapeutic range of the INR blood test. We found this to be true regardless of the self-reported frequency and variability with which foods containing high amounts of vitamin $\mathrm{K}$ were consumed.

Although it is a strength that our study participants (and their clinicians) were recruited from a geographically broad primary care population, baseline TTR for the group as a whole was slightly higher (mean, $72.2 \%)$ than that achieved in a nationally representative sample of Canadian primary care practices (mean, $67.8 \%) .{ }^{20}$ Conceivably, those who volunteered might be more compliant or healthier than average, with less opportunity for an intervention to demonstrate benefit. Our primary outcome is also a limitation in that patients with excellent baseline control can disproportionately drive it. Absolute change in TTR was, however, nearly identical in both groups, and we found literature-derived minimum clinically important differences that are substantially larger than the point estimates for both our primary outcome (observed change, $4.4 \%$; minimum clinically important difference

\section{Table 2. Anticoagulation Outcomes at 7 Months}

\begin{tabular}{|c|c|c|c|c|c|}
\hline Outcome & Morning & Evening & Difference & $P$ Value & $\begin{array}{l}95 \% \mathrm{Cl} \text { for } \\
\text { Difference }\end{array}$ \\
\hline \multicolumn{6}{|l|}{ Primary outcome } \\
\hline $\begin{array}{l}\text { Percent change in proportion of time outside } \\
\text { target INR range, median (IQR) }\end{array}$ & $\begin{array}{l}-11.9 \\
(-59.5 \text { to } 65.6)\end{array}$ & $\begin{array}{l}-22.9 \\
(-62.5 \text { to } 77.4)\end{array}$ & $4.4^{a}$ & .62 & -17.6 to 27.3 \\
\hline \multicolumn{6}{|l|}{ Secondary outcomes } \\
\hline Absolute change in TTR, \% & $2.9(22.1)$ & $3.0(22.7)$ & -0.1 & .97 & -6.1 to 5.9 \\
\hline Percent of patients with TTR $>75 \%$ & 56.0 & 54.6 & 1.4 & .89 & -12.3 to 14.9 \\
\hline Percent of patients with TTR $<60 \%$ & 22.0 & 22.2 & -0.2 & .99 & -11.5 to 11.9 \\
\hline Maximum out-of-range INR, median (IQR) & $3.6(3.3-4.0)$ & $3.6(3.4-4.0)$ & $0.0^{\mathrm{a}}$ & .52 & -0.2 to 0.1 \\
\hline Minimum out-of-range INR, median (IQR) & $1.6(1.3-1.8)$ & $1.7(1.5-1.8)$ & $-0.1^{\mathrm{a}}$ & .15 & -0.2 to 0.0 \\
\hline Percent of INR values above range, median (IQR) & $10.0(0-20.0)$ & $11.1(0-19.7)$ & $0.0^{\mathrm{a}}$ & .90 & -1.8 to 1.4 \\
\hline Percent of INR values below range, median (IQR) & $15.0(0-28.6)$ & $12.8(0-22.6)$ & $0.0^{\mathrm{a}}$ & .48 & -0.5 to 4.3 \\
\hline
\end{tabular}


$\pm 20 \%$ ), and the absolute change in TTR (observed change, $-0.1 \%$; minimum clinically important difference, $\pm 6 \%$ ). TTR is also limiting in that it is a surrogate outcome. Our study was not powered to examine differences in clinical events.

To our knowledge, although patient-facing information commonly suggests warfarin should be taken in the evening, ${ }^{6-10}$ there is no evidence to support this practice. Of 1,642 articles returned in our literature review (searching PubMed with the term warfarin $[\mathrm{MESH}]$ and filtering for clinical trials), none examined the influence of warfarin administration time. We believe our study is the first to address this question.

The lack of a dietary interaction, and the apparent absence of any effect of warfarin administration time on INR stability might be explained, at least in part, by the 36- to 42 -hour half-life of racemic warfarin. ${ }^{11}$ It is also possible that the benefit on TTR is real, but only observable with ultrashort testing intervals that weekly (or longer) INR testing would miss. If we assume true benefit equivalent to the 12 -hour lead time for dosing changes that evening (vs morning) administration might bring about, however, it would have very little effect on TTR. In our experience, patients seldom exceed 2 dose changes per month. If each (evening) dose change provided 12 extra hours in range, that would provide at best an extra 24 hours in range per month, a clinically unimportant improvement in TTR of $3.3 \%$ or less.

Although direct oral anticoagulants (DOACs) have begun replacing warfarin in recent years, warfarin is superior to DOACs, on both thromboembolic and bleeding outcomes, in patients with mechanical heart valves. ${ }^{21,22}$ Hence, it is likely to remain the anticoagulant of choice in that population. Given warfarin is also much less expensive than DOACs, and may provide similar outcomes in patients with TTR greater than $65 \%,{ }^{23,24}$ it will also remain a viable option when cost is a concern to patients, especially in countries where mean TTR exceeds 65\% (eg, Sweden, United Kingdom, Canada). ${ }^{20,25,26}$

We have found the percent change in time outside therapeutic INR range resulting from morning vs evening warfarin administration to be neither statistically significant nor clinically important should the observed difference be real. Despite common and widespread advice to the contrary, warfarin administration time does not appear to be clinically important. Given that patients and their community caregivers might find a particular time of day more convenient or easier to remember, warfarin administration time should be tailored to patient preference.

To read or post commentaries in response to this article, see it online at http://www.AnnFamMed.org/content/18/1/42.
Submitted January 19, 2019; submitted, revised, May 5, 2019; accepted June 12, 2019.

Key words: chronotherapy; warfarin; Coumadin; TTR; anticoagulation; atrial fibrillation; thromboembolism; mechanical valve; primary care; practice-based research

Funding support: Funding for this work came solely from nonprofit sources including the Vancouver Coastal Health Research Institute, the research arm of a regional health authority (https://www.vchri.ca/aboutus); the Richmond Hospital Foundation, a community hospital foundation (http://www.richmondhospitalfoundation.com/home); and EnACt, a government-funded organization fostering primary care research (https://primarycareresearch.ca/).

Disclaimer: The funders of the study had no role in study design, data collection, data analysis, data interpretation, writing of the paper, or the decision to submit for publication.

Previous presentation: This work was previously presented at the North American Primary Care Research Group (NAPCRG) 2018 Annual Meeting; November 12, 2018; Chicago, Illinois.

Acknowledgments: We would like to thank the many medical office assistants, and other primary care team members, who devoted time to preparing and mailing recruitment letters-in particular, the INR nurses of the Oliver Primary Care Network. We would also like to thank both Dorna Sadeghi, who assisted with data analysis, and the volunteer family physicians of the Pragmatic Trials Collaborative, who enabled this work to take place in their community practices.

Trial registration: NCT02376803

\section{References}

1. Albers GW, Sherman DG, Gress DR, Paulseth JE, Petersen P. Stroke prevention in nonvalvular atrial fibrillation: a review of prospective randomized trials. Ann Neurol. 1991;30(4):511-518.

2. Singer DE, Chang Y, Fang MC, et al. Should patient characteristics influence target anticoagulation intensity for stroke prevention in nonvalvular atrial fibrillation?: the ATRIA study. Circ Cardiovasc Qual Outcomes. 2009;2(4):297-304.

3. Hylek EM, Go AS, Chang Y, et al. Effect of intensity of oral anticoagulation on stroke severity and mortality in atrial fibrillation. N Engl J Med. 2003;349(11):1019-1026.

4. Wan Y, Heneghan C, Perera R, et al. Anticoagulation control and prediction of adverse events in patients with atrial fibrillation: a systematic review. Circ Cardiovasc Qual Outcomes. 2008;1(2):84-91.

5. White HD, Gruber M, Feyzi J, et al. Comparison of outcomes among patients randomized to warfarin therapy according to anticoagulant control: results from SPORTIF III and V. Arch Intern Med. 2007;167(3):239-245.

6. Cleveland Clinic. Anticoagulant Medication Warfarin (Coumadin). https://my.clevelandclinic.org/health/drugs/4713-anticoagulantmedication-warfarin-coumadin. Accessed Jan 25, 2018.

7. National Health Service (NHS). Warfarin. https://www.nhs.uk/medicines/warfarin/. Accessed Jan 25, 2018.

8. HealthLinkBC. Warfarin: taking your medicine safely. https://www. healthlinkbc.ca/health-topics/aba5406. Accessed Dec 18, 2019.

9. Pharmaceutical Management Agency of New Zealand (PHARMAC). Starting on Warfarin. http://www.bestpractice.net.nz/resources/AF/ PHARMAC_warfarin.pdf. Accessed Apr 21, 2018. 
10. Government of Western Australia, Department of Health. Living with warfarin. Information for patients. http://ww2.health.wa.gov. au/ /media/Files/Corporate/general\%20documents/Quality/PDF/ Living-with-Warfarin.pdf. Accessed Apr 21, 2018.

11. Hirsh J, Fuster V, Ansell J, Halperin JL; American Heart Association; American College of Cardiology Foundation. American Heart Association/American College of Cardiology Foundation guide to warfarin therapy. Circulation. 2003;107(12):1692-1711.

12. Olson RE. The function and metabolism of vitamin K. Annu Rev Nutr. 1984:4:281-337.

13. Hansson L, Hedner T, Dahlöf B. Prospective randomized open blinded end-point (PROBE) study. A novel design for intervention trials. Prospective Randomized Open Blinded End-Point. Blood Press. 1992;1(2):113-119.

14. Pragmatic Trials Collaborative. Measuring What Matters. http:// www.PragmaticTrials.ca/. Accessed March 14, 2018.

15. Heran BS, Allan GM, Green L, et al. Effect of medication timing on anticoagulation stability in users of warfarin (the INRange RCT): study protocol for a randomized controlled trial. Trials. 2016;17(1): 391.

16. Harris PA, Taylor R, Thielke R, Payne J, Gonzalez N, Conde JG. Research electronic data capture (REDCap)-a metadata-driven methodology and workflow process for providing translational research informatics support. J Biomed Inform. 2009;42(2):377-381.

17. Samsa GP, Matchar DB. Relationship between test frequency and outcomes of anticoagulation: a literature review and commentary with implications for the design of randomized trials of patient selfmanagement. J Thromb Thrombolysis. 2000;9(3):283-292.

18. Wilson SJ, Wells PS, Kovacs MJ, et al. Comparing the quality of oral anticoagulant management by anticoagulation clinics and by family physicians: a randomized controlled trial. CMAJ. 2003;169(4): 293-298.

19. Nieuwlaat R, Eikelboom JW, Schulman S, et al. Cluster randomized controlled trial of a simple warfarin maintenance dosing algorithm versus usual care among primary care practices. J Thromb Thrombolysis. 2014;37(4):435-442.
20. Liu S, Singer A, McAlister FA, et al. Quality of warfarin management in primary care: Determining the stability of international normalized ratios using a nationally representative prospective cohort. Can Fam Physician. 2019;65(6):416-425.

21. Eikelboom JW, Connolly SJ, Brueckmann M, et al.; RE-ALIGN Investigators. Dabigatran versus warfarin in patients with mechanical heart valves. N Engl J Med. 2013;369(13):1206-1214.

22. January CT, Wann LS, Calkins H, et al. 2019 AHA/ACC/HRS Focused update of the 2014 AHA/ACC/HRS Guideline for the Management of Patients With Atrial Fibrillation: a report of the American College of Cardiology/American Heart Association Task Force on Clinical Practice Guidelines and the Heart Rhythm Society in collaboration With the Society of Thoracic Surgeons. Circulation. 2019;140(2): e125-e151.

23. Gomez-Outes A, Terleira-Fernandez Al, Calvo-Rojas G, Suarez-Gea ML, Vargas-Castrillon E. Dabigatran, rivaroxaban, or apixaban versus warfarin in patients with nonvalvular atrial fibrillation: a systematic review and meta-analysis of subgroups. Thrombosis. 2013;2013: 640723.

24. Wallentin L, Yusuf S, Ezekowitz MD, et al.; RE-LY investigators. Efficacy and safety of dabigatran compared with warfarin at different levels of international normalised ratio control for stroke prevention in atrial fibrillation: an analysis of the RE-LY trial. Lancet. 2010; 376(9745):975-983.

25. Wieloch $M$, Själander A, Frykman V, Rosenqvist M, Eriksson N, Svensson PJ. Anticoagulation control in Sweden: reports of time in therapeutic range, major bleeding, and thrombo-embolic complications from the national quality registry AuriculA. Eur Heart J. 2011; 32(18):2282-2289.

26. Jones M, McEwan P, Morgan CL, Peters JR, Goodfellow J, Currie CJ. Evaluation of the pattern of treatment, level of anticoagulation control, and outcome of treatment with warfarin in patients with non-valvar atrial fibrillation: a record linkage study in a large British population. Heart. 2005;91(4):472-477. 